\title{
Down-regulation of MTUS1 in human colon tumors
}

\author{
CHRISTINA ZUERN ${ }^{1,3}$, JUTTA HEIMRICH ${ }^{1}$, ROLAND KAUFMANN ${ }^{2}$, KONRAD K. RICHTER $^{2}$, \\ UTZ SETTMACHER $^{2}$, CHRISTOPH WANNER ${ }^{1}$, JAN GALLE $^{1}$ and STEFAN SEIBOLD ${ }^{1}$ \\ ${ }^{1}$ Department of Medicine, Division of Nephrology, University Clinic of Wuerzburg, Wuerzburg; \\ ${ }^{2}$ University Hospital, Department of General and Visceral Surgery, Friedrich Schiller University, Jena; \\ ${ }^{3}$ Department of Nephrology and Hypertension, University of Erlangen-Nürnberg, Erlangen, Germany
}

Received July 14, 2009; Accepted September 3, 2009

DOI: $10.3892 /$ or_00000621

\begin{abstract}
Loss of proliferative control and failure to undergo cellular differentiation are key events during carcinogenesis. We recently identified a new potential tumor suppressor gene named MTUS1 (mitochondrial tumor suppressor 1), downregulated in undifferentiated tumor cell lines, inhibiting tumor cell proliferation after recombinant over-expression. The aim of this study was to investigate whether MTUS1 is also down-regulated in human tumor tissues, and whether reduced expression of MTUS1 enhances cellular proliferation. Expression of MTUS1 in human colon cancer tissues was compared with corresponding normal colon tissues using Western blot analysis and RT-PCR. Investigation of the DNA sequence and methylation pattern was performed using bisulfite reaction and DNA sequencing. Promotor activity was measured by promoter assays. Silencing of MTUS1 was carried out by siRNA transfection. Proliferation was measured by cell count. MTUS1 expression is significantly down-regulated in colon cancer tissues, compared to the corresponding normal tissues, on protein and mRNA level. No mutations of MTUS1 were detected in the coding sequence or the predicted promoter region in cancer tissues. No difference of $\mathrm{CpG}$ methylation, but an altered $\mathrm{CpNpG}$ methylation was found in the predicted promoter region. Functional significance of the predicted promoter region was demonstrated by promoter assays. Down-regulation of the MTUS1 expression by siRNA transfection significantly increased cellular proliferation. This study demonstrates a significant down-regulation of the MTUS1 expression in human colon cancer tissues. Since reduced expression of MTUS1 results in increased cellular proliferation, these data suggest that MTUS1 could be involved in the loss of proliferative control in human colon cancer.
\end{abstract}

Correspondence to: Dr Christina Zuern, Department of Nephrology and Hypertension, University of Erlangen-Nürnberg, Loschgestr. 8, D-91054 Erlangen, Germany

E-mail: christina.zuern@uk-erlangen.de

Key words: MTUS1, ATIP, tumor progression, colon cancer, 8p21.3-22, MTSG1, proliferation

\section{Introdution}

The multistage process of carcinogenesis includes the progressive acquisition of genetic alterations, resulting in activation of proto-oncogenes and inactivation of tumor suppressor genes. Consequential critical events in the evolution of tumors include the loss of proliferative control and the failure to undergo cellular differentiation. Genes located at chromosome 8p21.3-22 near marker D8S254 participate in tumor progression in colorectal cancer, pancreatic cancer, breast cancer, esophageal cancer, hepatocellular carcinoma, lung cancer, prostate cancer, urinary bladder carcinoma and head and neck squamous cell carcinoma (1-15). We previously identified a new gene named MTSG1, only $<0.9 \mathrm{Mb}$ apart from the D8S254 marker locus, regulating tumor cell proliferation (16). In accordance with the Guidelines for Human Gene Nomenclature it was recommended to change the gene name to MTUS1 (mitochondrial tumor suppressor 1), as it meanwhile appears in most databases. MTUS1 was discovered by investigating the molecular regulation during cellular transition from proliferation to senescence and differentiation in a three-dimensional collagen type I cell culture model. We thereby observed a transient up-regulation of MTUS1 during the initiation of a differentiated and senescent cellular phenotype in human endothelial cells. Additionally a reverse correlation of the MTUS1 mRNA expression with cellular proliferation and differentiation was noted in various pancreatic tumor cell lines, showing low expression in undifferentiated proliferating cell lines and high expression in differentiated and slowly proliferating cell lines (16). Further support for the hypothesis that MTUS1 might influence tumor progression by regulating tumor cell proliferation arose from studies in a pancreatic tumor cell line expressing no native MTUS1 mRNA, since recombinant over-expression of MTUS1 in this cell line resulted in significant inhibition of tumor cell proliferation (16). Previously, the inhibitory effect of MTUS1 on cell proliferation was linked to the growth inhibitory signaling cascade by trans-inactivation of receptor tyrosine kinases in insulin, bFGF, PDGF and EGF-induced ERK2 activation (17). A previously published investigation demonstrated a significant down-regulation of MTUS1 in ovarian carcinoma tissues, implicating a possible role of MTUS1 on the development of ovarian carcinoma (18). In addition, new studies revealed lower expression of MTUS1 in 
head and neck squamous cell carcinoma, down-regulation in colon polyps and copy number variant in breast cancer (19-21).

The gene responsible for tumor progression in colon cancer, located at chromosome 8p21.3-22 near marker D8S254, would be expected to be down-regulated in colon cancer $(1,10)$. Therefore, the aim of the present study was firstly to confirm down-regulation of MTUSI expression in colon cancer tissues, secondly to investigate the underlying genetic background responsible for the down-regulation of MTUS1, and thirdly to investigate whether down-regulation of MTUS1 results in increased cellular proliferation. This study supports the hypothesis that MTUS1 acts as a candidate tumor suppressor gene, responsible for tumor progression in human colon cancer.

\section{Materials and methods}

Tumor tissue acquisition. Colon tissue was obtained from ten unrelated patients admitted to the university hospital in Jena, Germany for the surgical removal of colon cancer. Small tissue parts from the tumor tissue itself, as well as from the surrounding normal non-tumor tissue, were removed for protein and total RNA isolation. The tissue sections were routinely investigated by the local pathologist. The local Ethics Committee approved the study, and all the patients gave their informed consent prior to surgery.

Generation of an MTUS1 specific polyclonal antibody. A polyclonal rabbit anti-MTUS1 antibody was established in collaboration with Eurogentec (Seraing, Belgium). Therefore, the peptide H2N-CPRNSGSFPSPSISPR-COOH (amino acids 422-436 of the MTUS1 protein sequence) was coupled to hemocyanin. Rabbits were immunized with the modified peptide on days $0,14,28$ and 56 , and the final bleed was carried out at day 80 of the immunization procedure. Subsequently the rabbit serum was affinity purified with the antigen peptide coupled to an AF-Amino Toyopearl $650 \mathrm{M}$ matrix (Tosoh Bioscience, Stuttgart, Germany).

Recombinant expression of MTUS1. Primers 5'-cgggggtaccgc ctcttctgtgaaatctcgec-3' and 5'-gcggatcctcatctgggtgaaatgctgg ggct-3' were used for RT-PCR. The PCR product was cloned into the pcDNA3 vector (Invitrogen, Karlsruhe, Germany) using the introduced $K p n I$ and BamHI restriction sites, and the correct sequence was confirmed by sequence analysis. MIA PaCa- 2 cells (ATCC, USA) $\left(10^{5}\right.$ cells/well) were transfected with $2 \mu \mathrm{g}$ plasmid DNA per 6-well using Lipofectamine Plus transfection reagent (Invitrogen) as recommended by the manufacturer. pcDNA3 vector alone was used as a control. Cells were harvested for protein isolation $24 \mathrm{~h}$ after transfection.

Western blot analysis. Proteins were isolated from tumor tissue, as well as from the corresponding normal colon tissue from the same patients. Therefore, tissues were homogenized and directly lysed in $50 \mathrm{mM}$ HEPES pH 7.5, $50 \mathrm{mM} \mathrm{NaCl}$, $20 \mathrm{mM}$ EDTA, $1 \mathrm{mM} \mathrm{MgCl}$, $2 \%$ Triton X-100, and protease inhibitor (complete mini, Roche Diagnostics, Mannheim, Germany). Proteins were isolated under the same conditions from cells, transfected with either pcDNA3 vector or siRNA. Subsequently, protein concentration was determined with the
Lowry reaction. Ten micrograms of protein were loaded per lane, transferred to a PVDF membrane (Amersham, Freiburg, Germany) and blocked with 5\% non-fat dried milk. Primary antibodies were polyclonal rabbit anti-MTUS1 (described above) diluted 1:2,000 in TBS-T containing 5\% non-fat dried milk, monoclonal mouse anti-PCNA (Transduction Laboratories, Lexington, KY, USA) diluted 1:2,000 in TBS-T containing 5\% non-fat dried milk, polyclonal goat anti-Ki-67 (Santa Cruz, Santa Cruz, USA) diluted 1:500 in TBS-T containing 5\% non-fat dried milk, and monoclonal mouse anti- $\beta$-actin (Sigma, Taufkirchen, Germany) diluted 1:20,000 in TBS-T containing 5\% non-fat dried milk. Secondary antibodies were HRP-conjugated goat anti-rabbit (Dako, Hamburg, Germany), goat anti-mouse (Dako), and mouse anti-goat (Sigma), all diluted 1:2000. For detection ECL Plus (Amersham) was used.

Tumor tissue mRNA expression. Total RNA isolation from tumor tissue and corresponding normal surrounding tissue was performed using the RNeasy Mini Kit (Qiagen, Hilden, Germany) as recommended by the manufacturer. First strand cDNA synthesis was performed at $37^{\circ} \mathrm{C}$ for $1 \mathrm{~h}$ applying $1 \mu \mathrm{g}$ of total RNA using oligo-dT primers. Two microliters of the cDNA reaction were subsequently applied in a multiplex PCR with 5'-gcctcttctgtgaaatctcgec-3' and 5'-cgctcagacagcag gtgctg-3' as MTUS1 specific primers and 5'-tctacaatgagctgcgt gtg-3' and 5'-tacatggetggggtgttgaa-3' as $\beta$-actin specific primers $\left(35\right.$ cycles, $57^{\circ} \mathrm{C}$ annealing temperature). Multiplex RT-PCR was established testing various cycles to ensure absence of a plateau phase (data not shown). PCR products were separated on $2 \%$ agarose gels, and the fluorescence intensity of the appropriate bands was detected and evaluated with the Quantity One ${ }^{\circledR}$ quantization software (Bio-Rad, Munich, Germany).

Sequence analysis of the colon tumor tissue. Cytoplasmatic RNA of two colon tumor tissues was isolated and cDNA synthesis was performed as described above. For sequence analysis $1 \mu \mathrm{l}$ cDNA was amplified using 5'-gcagcttctagac ctgcaggagggag-3' and 5'-gagaacaacttggaagcgtgagcaga-3' as MTUS1-specific primers $\left(66,5^{\circ} \mathrm{C}\right.$ annealing temperature, 38 cycles), generating a DNA fragment containing the sequence of the 10 exons of MTUS1 (16). These PCR products were separated on a $1 \%$ agarose gel, bands were subsequently isolated using the Gel extraction kit (Qiagen) and the DNA was repeatedly sequenced (MWG, Ebersberg, Germany) with the reverse primer 5'-cgctcagacagcaggtgctg-3' and the following forward primers: 5'-agtggatttatcetgcagct-3', 5'-agtcgaaagtcaaca agcga-3', 5'-ctcagggccctattaagatt-3', 5'-cttacccatgtgagcctaac-3', 5'-ggagtccctttgtctttggg-3', 5'-gaagctagccactcagagaa-3', 5'ctgctcacgettccaagttg-3'.

For the analysis of the predicted promoter region the MTUS1-specific primers 5'-ctttgggaggccaaagcaggtggat-3' and 5'-gaagcagagcctgtgttaggatgtc-3' were amplified with genomic DNA $\left(60.5^{\circ} \mathrm{C}\right.$ annealing temperature, 38 cycles). The PCR products were separated on a $2 \%$ agarose gel, bands were isolated, and the DNA was sequenced (MWG Biotech).

Sequence analysis of the MiaPaCa-2 cells. DNA of MiaPaCa-2 cells was isolated using the DNA Tissue Kit (Qiagen). Respec- 
tively, exon sequences were amplified with $200 \mathrm{ng}$ of DNA using $M T U S 1$-specific primers $\left(60^{\circ} \mathrm{C}\right.$ annealing temperature, 36 cycles). For exon 1 forward primer 5'-cctgagaccacccactt tct-3' and reverse primer 5'-catcccccgtgcaacactaa 3' were used, for exon 2 the primers $5^{\prime}$-tccaaccatctagatctccg-3' and ccgacctcaagtagaaaggc-3', for exon 3 the primers $5^{\prime}$-cttcccaata ctgggtctca-3' and 5'-ataccagacagggagtgcca-3', for exon 4 the primers 5'-gcttcaggtgtcatcacagc-3' and 5'-caagtaagcagcagagg cct-3', for exon 5 the primers 5'-ccacgcttacctgtgaagct-3' and $5^{\prime}$-acagggcattcgctgaagca-3', for exons 6 and 7 the primers 5'-cggcttcatcatcaaccct-3' and 5'-ttctctggctgctgagtact-3', for exon 8 the primers 5'-gatgaacatctgtcgcttgc-3' and 5'-gcagttaac ccaactccaca-3', for exon 9 the primers 5 '-gtgcttgtgtcgtgaggtgc-3 and 5'-agagcgtgtccagaaagtac-3' and for exon 10 the primers 5'-cctgtgaccttgtgcaggat-3' and 5'-tactgtgctgtcacagggct-3' were used. For the predicted promoter region the specific primers 5'-ctttgggaggccaaagcaggtggat-3' and 5'-gaagcagagcctgtgttagg atgtc- $3^{\prime}$ were used $\left(60.5^{\circ} \mathrm{C}\right.$ annealing temperature, 38 cycles $)$. The PCR products were separated on $2 \%$ agarose gels, bands were isolated and sequenced with the respective forward primer (MWG Biotech).

Promoter assay. The program TESS of the Penn Centres for Bioinformatics predicted the MTUS1 promoter region in the range of position 4860-5100 in the Genbank sequence AF165145. Genomic DNA from HUVEC cells was isolated and two different constructs of the promoter region were manufactured. On the one hand the predicted promoter region and the following intron sequence was amplified $\left(60.7^{\circ} \mathrm{C}\right.$ annealing temperature, 38 cycles) using the specific forward primer 5'-ccctcgagggagagcagtctgatgt-3' and on the other hand the intron region alone was amplified using the specific forward primer 5'-ccetcgaggtggaaaaattgggect-3' (both primers including an $\mathrm{XhoI}$ restriction site). For both constructs 5'-gga agcttcactttcagatgttgcca-3' (including a HindIII restriction site) was used as a reverse primer. Both fragments were cloned into the XhoI and HindIII restriction sites into the pGl3 basic vector (Promega, Mannheim, Germany) and the correct construct insertion was confirmed by sequence analysis.

MiaPaCa- 2 cells were grown at $37^{\circ} \mathrm{C}, 5 \% \mathrm{CO}_{2}$, in DMEM supplemented with $10 \%$ fetal bovine serum (FBS) and $2 \mathrm{mM}$ glutamine. For the reporter assay $1 \times 10^{4}$ cells were seeded per well on a 96-well plate and the cells were transfected with $0.5 \mu \mathrm{g}$ of the different plasmid DNAs using GenePORTER $2^{\mathrm{TM}}$ (Gene Therapy Systems, San Diego, CA, USA) according to the manufacturer's instructions. Luciferase assays were carried out with the Dual Luciferase Reporter Assay (Promega) as described by the manufacturer, using Renilla luciferase (pRL-TK vector) as a control for transfection efficiency.

Analysis of DNA methylation of the predicted promoter region. Genomic DNA was isolated from HUVEC and MiaPaCa-2 cells, four colon tumors and corresponding normal tissues with the DNA Tissue Kit (Qiagen). Approximately $1 \mu \mathrm{g}$ of DNA was modified by bisulfite reaction as described earlier (22).The following PCR reaction of the bisulfite-modified DNA was performed with the specific primers 5 '-gtttatgttaagt ttaaaaataaattggagagt-3' (upstream) and 5'-tcaatacaaaaataaatttt aattatcaaaaaa-3' (downstream), $45^{\circ} \mathrm{C}$ annealing temperature and 38 cycles. PCR products were purified with the PCR

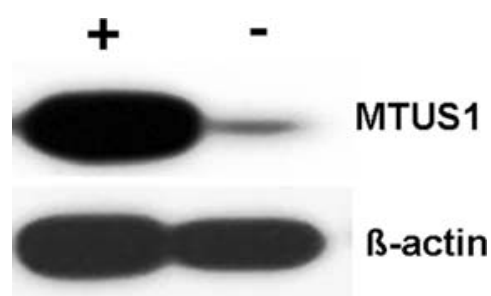

Figure 1. Western blot analysis demonstrating the correct detection of the $50 \mathrm{kDa}$ MTUS1 protein, using the affinity-purified polyclonal rabbit antiMTUS1 antibody directed against the peptide H2N-CPRNSGSFPSPSISPR$\mathrm{COOH}$ (amino acids $422-436$ of the MTUS1 protein sequence). + , MiaPaCa cells transfected with recombinant MTUS1 protein. -, Control MiaPaCa2 cells transfected with the pcDNA3 vector only. B-actin staining was used to ensure equal loading of the proteins.

Purification Kit (Qiagen) and a nested PCR reaction $\left(49^{\circ} \mathrm{C}\right.$ annealing temperature, 38 cycles) was performed with the specific primers 5'-ttaaagtaggtggattataaggttaggatattg-3' (upstream) and 5'-acaataaacaaaataaaaatttaaattaaaaac-3' (downstream). PCR products were loaded on a $2 \%$ agarose gel, isolated and the DNA was sequenced with the specific forward primer 5'-taaggttaggatattgagattattttggttaat-3'.

Down-regulation of MTUS1 expression by siRNA transfection. We used double-stranded siRNA (MWG, Ebersberg, Germany), directed against the sequence 5'- agccaggaatgagtta caa-3', for silencing of the MTUS1 gene. For transfection, HUVEC $\left(1 \times 10^{5}\right.$ per 6 -well) were incubated with GenePORTER 2 (Gene Therapy Systems) $(2.5 \mu \mathrm{l} / \mathrm{ml}$ medium) and various concentrations of siRNA $(10-50 \mathrm{nM})$ for $4 \mathrm{~h}$, according to the manufacturer's recommendations. Thereafter, $1 \mathrm{ml}$ of growth medium was added. Twenty-four hours after transfection cells were washed with PBS, and harvested for protein isolation. Luciferase siRNA (MWG) transfected cells were used as a control.

Proliferation assay. HUVEC ( $1 \times 10^{5}$ per 6 -well) were transfected with $25 \mathrm{nM}$ MTUS1 siRNA, using GenePORTER 2 (2.5 $\mu \mathrm{l} / \mathrm{ml}$ medium) according to the manufacturer's recommendations. Four hours after transfection $1 \mathrm{ml}$ of growth medium was added. Forty-eight hours after transfection, cells were harvested and counted using a Coulter Z2 (Beckman Coulter, Krefeld, Germany). Luciferase siRNA (MWG) transfected cells were used as a control.

Statistical analyses. Data are presented as mean \pm SEM and analyzed by ANOVA or Student's t-test. A $\mathrm{P}<0.05$ was considered significant.

\section{Results}

Characterization of the MTUS1 antibody. After affinity purification of the MTUS1 antibody, the final quality control showed $>85 \%$ purity in SDS-PAGE and a high specificity in ELISA (data not shown). Western blot analysis confirmed the sensitivity and specificity of the MTUS1 antibody, demonstrating detection of the MTUS1 protein (50 kDa) (Fig. 1), and inhibition of the MTUS1 signal by addition of the blocking peptide (data not shown). 
MTUS protein expression is down-regulated in human colon cancer. We compared the MTUS1 protein expression in human colon cancer tissue with the MTUS1 protein expression in the corresponding normal surrounding colon tissue in each patient using Western blot analysis. We observed a $>25 \%$ down-regulation of the MTUS1 protein expression in the tumor tissue as compared to the corresponding normal surrounding tissue in 5 out of 10 patients (Fig. 2). The mean MTUS1 protein expression in the tumor tissues of these 5 patients was $44.8 \%$ (range 25.2-63.6\%) compared to normal colon tissue. The tumor samples of the other 5 patients investigated showed no significant down-regulation of the MTUS1 protein expression level (mean expression 92.8\%; range $82.1-107.4 \%$ ), compared to the normal surrounding colon tissue (data not shown). The absolute expression levels of the MTUS1 protein varied clearly between different patients.

The observed expression of the proliferation marker PCNA confirms a higher proliferative index in the tumor tissue compared to the corresponding normal tissue. Fig. 2A reveals a negative correlation between the MTUS1 and PCNA protein expression. The higher $\mathrm{Ki}-67$ protein expression in each tumor tissue compared to the corresponding normal tissue of the same patient confirms the correct assignment of the tissues to the 'tumor' and 'normal' category and presents a prognostic marker for overall and disease-free survival in colon cancer (23). The $B$-actin protein expression was determined in order to adjust the MTUS1 protein expression to the amount of total protein loaded per lane (Fig. 2).

MTUS mRNA expression is down-regulated in human colon cancer. We next investigated whether significant downregulation of the MTUS1 protein expression results from reduced MTUS1 mRNA expression, by comparing the MTUS1 mRNA expression in the colon cancer tissue with the respective normal surrounding tissue. We established a multiplex RT-PCR analysis to adequately adjust the MTUSI mRNA expression to the simultaneously obtained $\beta$-actin mRNA expression (Fig. 3A). In all patients with significant down-regulation of the MTUS1 protein expression, we observed a significant down-regulation of the MTUS1 mRNA expression in the colon cancer tissues as compared to their corresponding normal colon tissue (Fig. 3B). The mean MTUS1 mRNA expression in the tumor tissues was $46.4 \%$ (range 36.7-53.3\%) compared to the normal colon tissue.

MTUS1 down-regulation is not a consequence of mutations. To analyze the reasons for the down-regulation of MTUS1 in colon tumor tissue and different pancreatic tumor cell lines (16), sequence analysis were carried out on tumor tissues and the MiaPaCa-2 cell line. The exon sequencing of the MiaPaca- 2 cells revealed no relevant mutation in the coding sequence of MTUS1 and in the predicted promoter sequence. Also colon tumor tissues displayed no mutations in the coding sequence of MTUS1 or in the sequence of the predicted promoter region. Therefore, mutations in the exon sequence or the predicted promoter region are unlikely the cause for the low MTUS1 expression in MiaPaCa- 2 cells and colon tumor tissue.
A

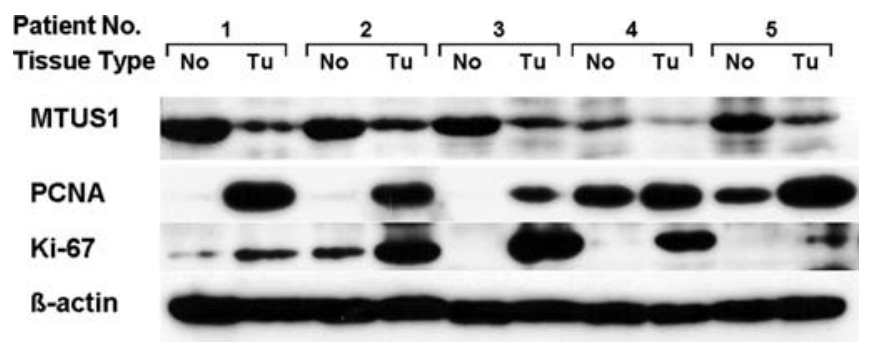

B

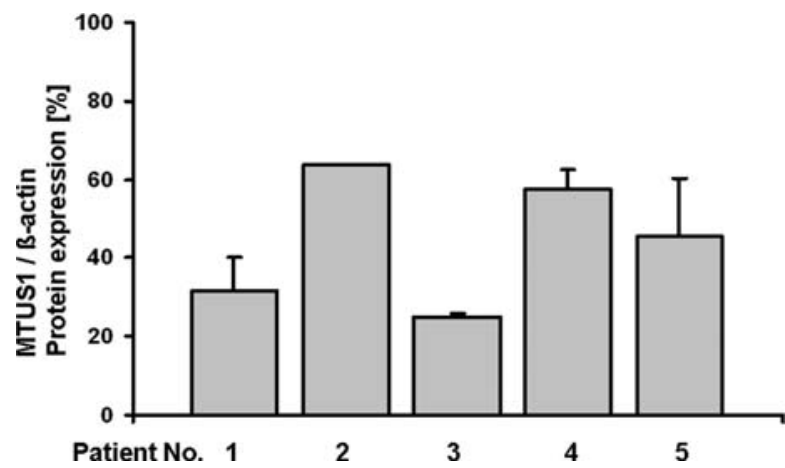

Figure 2. Western blot analysis comparing the protein expression of MTUS1, $P C N A$, and $K i-67$, in tumor tissue (Tu) and corresponding normal surrounding tissue (No) in five individual patients (nos. 1-5) suffering from colon cancer. (A) The blots shown are representative of at least three different experiments. B-actin staining was used to ensure equal loading of the proteins. (B) Graphical analysis of the MTUS1 protein expression. Results were adjusted to B-actin expression levels. MTUS1 expression in the normal surrounding tissue was defined as $100 \%$ in each individual patient. Data are mean \pm SEM from three independent experiments.

A

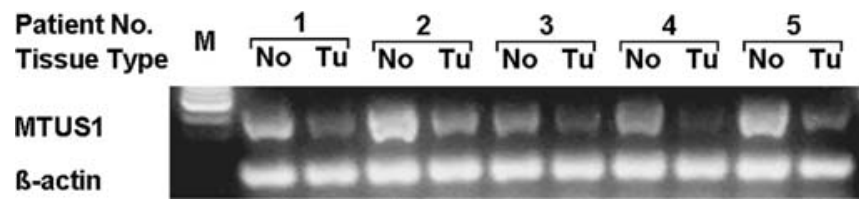

B

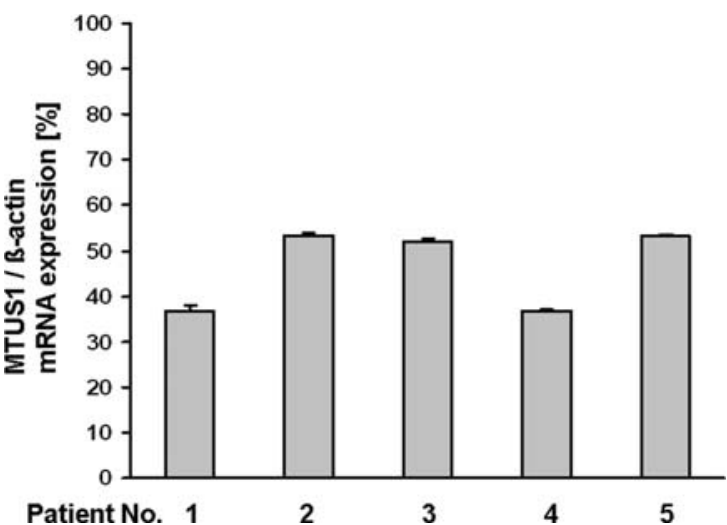

Figure 3. Multiplex RT-PCR analysis comparing the MTUS1 mRNA expression in tumor (Tu) and corresponding normal surrounding tissue (No) in the 5 patients (nos. 1-5) suffering from colon adenocarcinoma with $>25 \%$ down-regulation of the MTUS1 protein in the tumor tissue compared to the corresponding normal tissue. (A) Agarose gel of the multiplex RT-PCR. B-actin RT-PCR was performed to adjust template mRNA application. M, marker (B) Graphical analysis of the MTUS1 mRNA expression. Results were adjusted to $ß$-actin expression levels. MTUS1 expression in the normal surrounding tissue was defined as $100 \%$ in each patient. Data are mean \pm SEM from six independent experiments. 


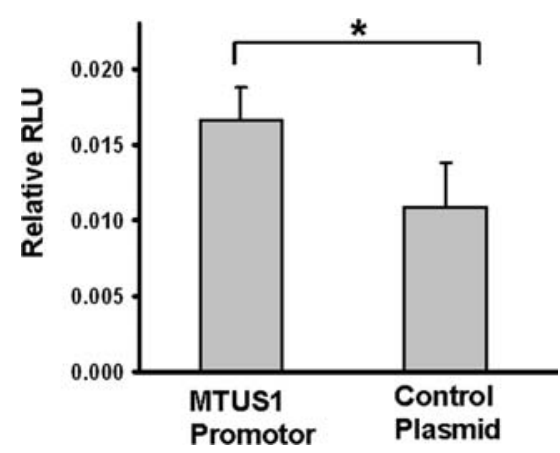

Figure 4. Promoter assay of MiaPaCA-2 cells transfected with either $0.5 \mu \mathrm{g}$ pGL3 vector DNA with an insert including the promoter range or $0.5 \mu \mathrm{g}$ vector DNA excluding the promoter range. Twenty-four hours after transfection, luminescence was measured using a Victor luminometer. Data are mean \pm SEM from five independent experiments.

The predicted promoter sequence regulates transcription of MTUS1. To substantiate that the analyzed promoter region executes transcriptional regulatory properties, we performed a promoter assay to test the promoter activity of two different constructs, including or excluding the predicted promoter area. The MiaPaCa-2 cells transfected with the promoter construct thereby revealed a significantly $(\mathrm{P}=0.018)$ higher luminescence (RLU 0.0166 \pm 0.0011 ) as compared to the control transfected cells $(0.0109 \pm 0.0015)$ (Fig. 4). This investigation therefore approved the hypothesis that the examined promoter sequence displays regulatory properties for MTUS1 transcription.

Variable DNA methylation of the predicted promoter sequence. We next investigated whether methylation of the MTUS1 promoter sequence could cause the down-regulation of the MTUS1 expression in tumor cells. Therefore bisulfite reactions were performed with DNA of MiaPaCa- 2 cells, of HUVEC cells and of four colon tumors and corresponding normal tissues. The analysis regarding the methylation pattern of the $\mathrm{CpG}$ and $\mathrm{CpNpG}$ islands did not reveal differences in the methylation of the $\mathrm{CpG}$ islands, but a clear hypomethylation of the $\mathrm{CpNpG}$ islands of the MiaPaCa-2 cells (100\%) in contrast to the HUVEC cells $(82.1 \%)$. In the colon tissue, the methylation pattern of the $\mathrm{CpG}$ islands also showed no differences between tumor and normal tissue. But as already seen in the MiaPaCa-2 cells, hypomethylation of the $\mathrm{CpNpG}$ islands was observed in the tumor tissues (mean of unmethylated cytosines $94.7 \%$, range $92.9-100 \%$ ) compared to the corresponding normal tissue (mean 67\%, range 28.6-89.3\%) (Fig. 5).

MTUS1 down-regulation results in increased cellular proliferation. In order to provide further evidence that MTUS1 plays an active role and is not simply a bystander of tumor development, we investigated the impact of MTUS1 downregulation on cellular proliferation using MTUS1 silencing by transfection of specific siRNA. Transfection efficacy of the cells was $85 \%$, as measured by FACS analysis using fluorescence-labeled siRNA (data not shown). Compared to luciferase transfected controls, cells transfected with $25 \mathrm{nM}$ of MTUS1 siRNA showed a $58 \pm 4 \%$ reduction in MTUS1

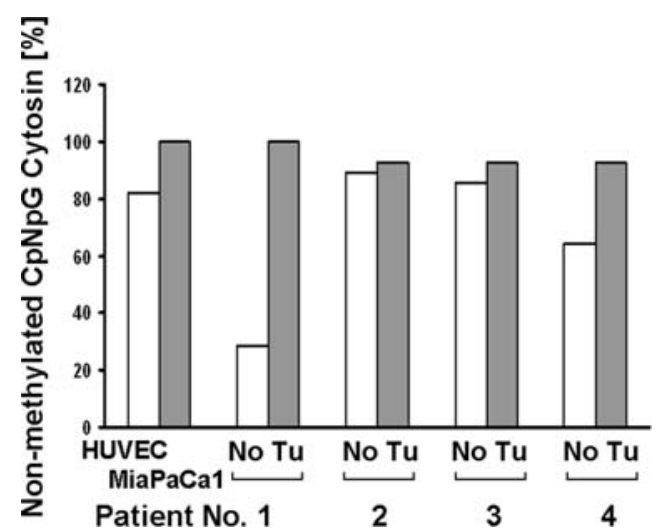

Figure 5. Methylation pattern of all $\mathrm{CpNpG}$ cytosines in the predicted promoter region of HUVEC cells, MiaPaCa-2 cells and four normal tissues (No) with corresponding colon tumor tissues (Tu).
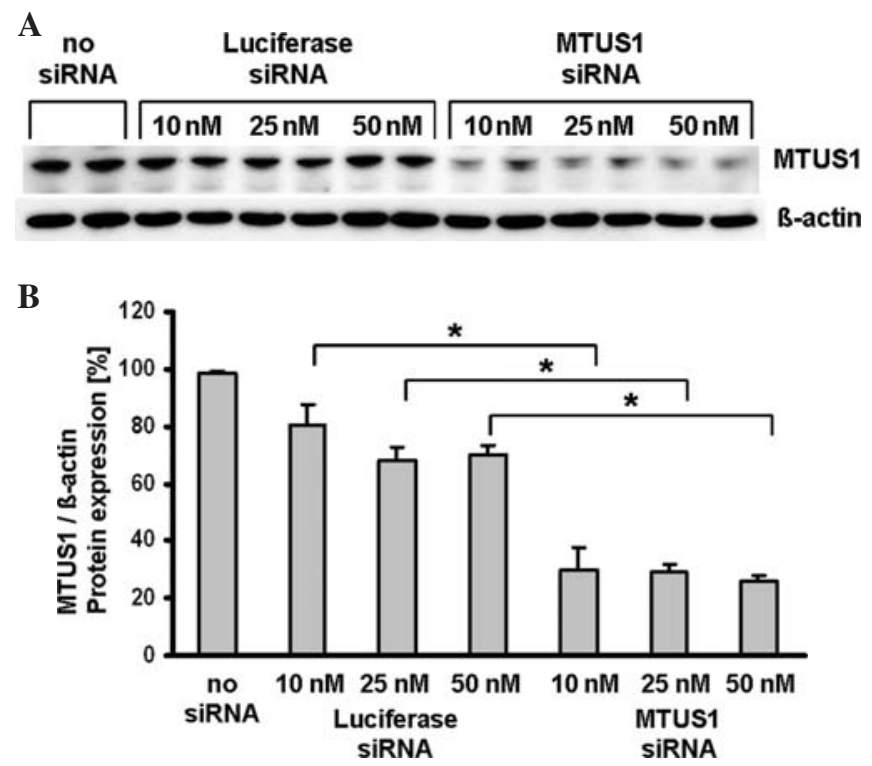

Figure 6. Western blot analysis comparing the MTUS1 protein expression in HUVEC transfected with various concentrations (10-50 nM) of either MTUS1 siRNA or Luciferase siRNA as control. B-actin staining was used to ensure equal loading of the proteins. (A) The blot shown is representative for three independent experiments. (B) Graphical analysis of the MTUS1 protein expression. Results were adjusted to $B$-actin expression levels. Data are mean \pm SEM from three independent experiments.

protein expression (Fig. 6). Similar degrees of MTUS1 protein down-regulation were obtained using 10 and $50 \mathrm{nM}$ of MTUS1 siRNA respectively (Fig. 6). Transfection of cells with $25 \mathrm{nM}$ of MTUS1 siRNA resulted in a significantly increased total cell number $(438,000 \pm 22,000)$ after 48 -h incubation with growth medium compared to luciferase transfected controls $(388,000 \pm 16,000)$ (Fig. 7). Thus, the relative difference of cells between both groups was $17 \%(\mathrm{P}=0.02)$.

\section{Discussion}

In this study we discovered a significant down-regulation of MTUS1 in patients suffering from colon cancer, and an increased cellular proliferation resulting from reduced MTUS1 expression. Thus, taking these results into consideration 


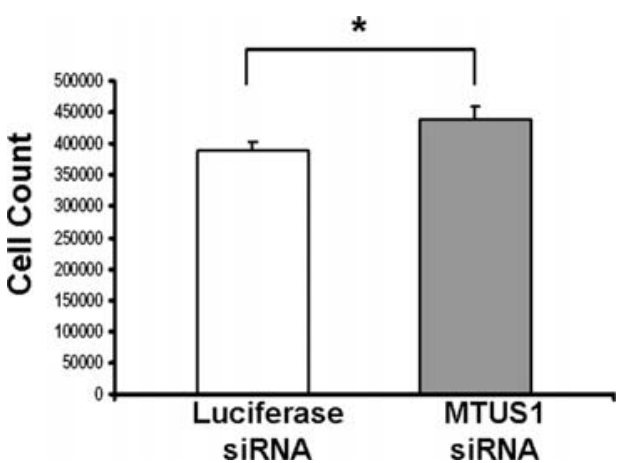

Figure 7. Proliferation assay of HUVEC $\left(1 \times 10^{5}\right.$ per 6 -well) transfected with either $25 \mathrm{nM}$ MTUS1 siRNA or $25 \mathrm{nM}$ Luciferase siRNA as control. Forty-eight hours after transfection, cells were harvested and counted using a Coulter Z2. Data are mean \pm SEM from four independent experiments.

Table I. Histological type, grading and pTNM classification of the colon carcinomas from patients with $>25 \%$ downregulation of MTUS1.

\begin{tabular}{lc}
\hline Patient no. & Tumor classification \\
\hline 1 & Adenocarcinoma G1 pT2N0M0 \\
2 & Adenocarcinoma G3 pTxNxMx \\
3 & Adenocarcinoma G3 pT4N2M1 \\
4 & Adenocarcinoma G2 pT3N0M0 \\
5 & Adenocarcinoma G1 pT1NxMx \\
\hline
\end{tabular}

together with previously published data, there are now several lines of evidence supporting the hypothesis that MTUS1 is involved in the regulation of tumor progression in various malignant diseases including human colon carcinoma: 1), MTUS1 is located at chromosome 8p21.3-22 near marker D8S254, exactly where the presence of genes involved in tumor progression was recorded. In colon cancer, genes at this chromosomal localization are thought to be responsible for progression of the malignant disease in $44 \%$ of patients (1), concordant with the observed down-regulation of MTUS1 in $50 \%$ of the investigated patients. Furthermore, this chromosomal region is also associated with disease progression in breast cancer, esophageal cancer, hepatocellular carcinoma, pancreatic cancer, lung cancer, prostate cancer, urinary bladder carcinoma, and head and neck squamous cell carcinoma (2-9). 2), monochromosome 8 transfer into colorectal cancer cell lines reduced their tumorigenicity and the metastatic potential in prostate tumor cells, confirming tumor suppressor activity of chromosome 8 in malignant diseases (24,25). 3), we observed an up-regulation of MTUS1 during the initiation of a differentiated and quiescent cellular phenotype, and we observed a reverse correlation of the MTUS1 expression with cellular proliferation and differentiation in different tumor cell lines (16). 4), and of particular importance, the tumor suppressor potential of MTUS1 was confirmed by overexpression of MTUS1 in a tumor cell line expressing no native MTUS1 mRNA, resulting in significantly reduced tumor cell proliferation (16). 5), the study presented here now further supports the hypothesis that MTUS1 is involved in tumor progression in human colon cancer, since we observed a significant down-regulation of the MTUS1 expression in $50 \%$ of patients suffering from colon carcinomas, as expected for the responsible gene at $8 \mathrm{p} 21.3-22(1) .6$ ), in this study we showed that down-regulation of MTUS1 resulted in increased cellular proliferation.

Taken together, gain of function experiments, as well as loss of function experiments clearly support the hypothesis that MTUS1 regulates cellular proliferation. The exact mechanism by which MTUS1 controls cellular proliferation is currently unknown, but MTUS1 is believed to be an early component of a growth inhibitory signaling pathway. It was shown that MTUS1 inhibits ERK2 activation and proliferation in eukaryotic cells stimulated with growth factors such as insulin, bFGF, PDGF and EGF, and it was suspected that MTUS1 inhibits cell proliferation via trans-inactivation of receptor tyrosine kinases (RTK) (17). Since members of the RTK signaling pathway such as EGFR, FGFR, PDGFB, RAS, RAF are well known to be involved in carcinogenesis, it is also well conceivable that MTUS1 is involved in tumor progression (26).

We herein confirmed the expected down-regulation of the MTUS1 expression in human colon cancer tissue. The mechanisms responsible for down-regulation of MTUS1 in human colon cancer and as previously reported in pancreatic tumor cell lines (16) seem not to be due to mutations in the coding region or the predicted promoter region. Our results of the sequencing analysis are also confirmed by a published investigation of $109 \mathrm{HCC}$ tumors and cell lines, where MTUS1 was not frequently mutated (27). However, the predicted promoter range was proved to have regulatory effects on the transcription and exhibited variable methylation patterns of the tumor tissue and cells compared to the normal tissue and cells. The $\mathrm{CpNpG}$ islands showed a hypomethylation in the MiaPaCa-2 cells and the tissues with down-regulated MTUS1 expression, while the $\mathrm{CpG}$ islands did not reveal any differences in methylation. Since it already has been reported that $\mathrm{CpNpG}$ methylation can occur in vivo and that methylation of these islands is suspected to stabilize the DNA (28), the hypomethylation of $\mathrm{CpNpG}$ islands could be a mechanism for the MTUS1 inactivation. Nevertheless, some published investigations have found deletion and some small mutations in the exon region of MTUS1, so that further analyses are needed to understand the mechanism of down-regulation in this gene $(19,21)$.

The histological type, grading and pTNM classification of the colon carcinomas (Table I) presumes that down-regulation of the MTUS1 expression can be an early event in malignant diseases, since also T1 and T2 tumors showed significant down-regulation of MTUS1.

\section{Acknowledgements}

The skillful technical assistance of Mrs. Elke Baumeister, Mrs. Carmen Bauer, and Mrs. Margarete Roeder is gratefully acknowledged. We would like to thank Dr Stepan Gambaryan from the Institute of Biochemistry and Pathobiochemistry at the University of Wuerzburg for helpful discussions. 


\section{References}

1. Fujiwara Y, Emi M, Ohata H, et al: Evidence for the presence of two tumor suppressor genes on chromosome $8 \mathrm{p}$ for colorectal carcinoma. Cancer Res 53: 1172-1174, 1993.

2. Bardi G, Johansson B, Pandis N, et al: Karyotypic abnormalities in tumours of the pancreas. Br J Cancer 67: 1106-1112, 1993.

3. Kerangueven F, Noguchi T, Coulier F, et al: Genome-wide search for loss of heterozygosity shows extensive genetic diversity of human breast carcinomas. Cancer Res 57: 5469-5474, 1997.

4. Ishii H, Baffa R, Numata SI, et al: The FEZ1 gene at chromosome 8p22 encodes a leucine-zipper protein, and its expression is altered in multiple human tumors. Proc Natl Acad Sci USA 96: 3928-3933, 1999.

5. Chan KL, Lee JM, Guan XY, Fan ST and Ng IO: High-density allelotyping of chromosome 8p in hepatocellular carcinoma and clinicopathologic correlation. Cancer 94: 3179-3185, 2002.

6. Wistuba II, Behrens C, Virmani AK, et al: Allelic losses at chromosome 8p21-23 are early and frequent events in the pathogenesis of lung cancer. Cancer Res 59: 1973-1979, 1999.

7. Suzuki H, Emi M, Komiya A, et al: Localization of a tumor suppressor gene associated with progression of human prostate cancer within a 1.2 Mb region of 8p22-p21.3. Genes Chromosomes Cancer 13: 168-174, 1995.

8. Wagner U, Bubendorf L, Gasser TC, et al: Chromosome 8p deletions are associated with invasive tumor growth in urinary bladder cancer. Am J Pathol 151: 753-759, 1997.

9. El Naggar AK, Coombes MM, Batsakis JG, Hong WK, Goepfert H and Kagan J: Localization of chromosome 8p regions involved in early tumorigenesis of oral and laryngeal squamous carcinoma. Oncogene 16: 2983-2987, 1998.

10. Tsai MH, Yang YC, Chen KH, et al: RER and LOH association with sporadic colorectal cancer in Taiwanese patients. Hepatogastroenterology 49: 672-677, 2002.

11. Vocke CD, Pozzatti RO, Bostwick DG, et al: Analysis of 99 microdissected prostate carcinomas reveals a high frequency of allelic loss on chromosome 8p12-21. Cancer Res 56: 2411-2416, 1996.

12. Yaremko ML, Recant WM and Westbrook CA: Loss of heterozygosity from the short arm of chromosome 8 is an early event in breast cancers. Genes Chromosomes Cancer 13: 186-191, 1995.

13. Chuaqui RF, Sanz-Ortega J, Vocke C, et al: Loss of heterozygosity on the short arm of chromosome 8 in male breast carcinomas. Cancer Res 55: 4995-4998, 1995.

14. El Naggar AK, Mao L, Staerkel G, et al: Genetic heterogeneity in saliva from patients with oral squamous carcinomas: implications in molecular diagnosis and screening. J Mol Diagn 3: 164-170, 2001.
15. Sasiadek M, Stembalska-Kozlowska A, Smigiel R, Krecicki T, Blin N and Mirghomizadeh F: Microsatellite and chromosome instability in squamous cell laryngeal carcinoma. Int J Oncol 19: 401-405, 2001.

16. Seibold S, Rudroff C, Weber M, Galle J, Wanner C and Marx M: Identification of a new tumor suppressor gene located at chromosome 8p21.3-22. FASEB J 17: 1180-1182, 2003.

17. Nouet S, Amzallag N, Li JM, et al: Trans-inactivation of receptor tyrosine kinases by novel angiotensin II AT2 receptorinteracting protein, ATIP. J Biol Chem 279: 28989-28997, 2004.

18. Pils D, Horak P, Gleiss A, et al: Five genes from chromosomal band 8 p22 are significantely down-regulated in ovarian carcinoma: N33 and EFA6R have potential impact on overall survival. Cancer 104: 2417-2429, 2005.

19. Ye H, Pungpravat N, Huang B, et al: Genomic assessments of the frequent $\mathrm{LOH}$ region on 8p22-p21.3 in head and neck squamous cell carcinoma. Cancer Genet Cytogenet 176: 100-106, 2007.

20. Lee S, Bang S, Song K and Lee I: Differential expression in normal-adenoma-carcinoma sequence suggests complex molecular carcinogenesis in colon. Oncol Rep 16: 747-754, 2006.

21. Frank B, Bermejo JL, Hemminki K, et al: Copy number variant in the candidate tumor suppressor gene MTUS1 and familial breast cancer risk. Carcinogenesis 28: 1442-1445, 2007.

22. Raizis AM, Schmitt F and Jost JP: A bisulfite method of 5methylcytosine mapping that minimizes template degradation. Anal Biochem 226: 161-166, 1995.

23. Garrity MM, Burgart LJ, Mahoney MR, et al: Prognostic value of proliferation, apoptosis, defective DNA mismatch repair, and p53 overexpression in patients with resected Dukes' B2 or C colon cancer: a North Central Cancer Treatment Group Study. J Clin Oncol 22: 1572-1582, 2004.

24. Gustafson CE, Wilson PJ, Lukeis R, et al: Functional evidence for a colorectal cancer tumor suppressor gene at chromosome 8p2223 by monochromosome transfer. Cancer Res 56: 5238-5245, 1996.

25. Ichikawa T, Nihei N, Suzuki H, et al: Suppression of metastasis of rat prostatic cancer by introducing human chromosome 8 . Cancer Res 54: 2299-2302, 1994.

26. Vogelstein B and Kinzler KW: Cancer genes and the pathways they control. Nat Med 10: 789-799, 2004.

27. Di Benedetto M, Pinneau P, Nouet S, et al: Mutation analysis of the $8 \mathrm{p} 22$ candidate tumor suppressor gene ATIP/MTUS1 in hepatocellular carcinoma. Mol Cell Endocrinol 252: 207-215, 2006.

28. Lorincz MC and Groudine $\mathrm{M}: \mathrm{CmC}(\mathrm{a} / \mathrm{t}) \mathrm{GG}$ methylation: a new epigenetic mark in mammalian cells? Proc Natl Acad Sci USA 98: 10034-10036, 2001. 